\title{
Cambiando paradigmas en el diagnóstico (primario y de las recidivas) y en el tratamiento del Cáncer Urotelial: preparando el futuro
}

\author{
Antoni Gelabert Mas \\ Servicio y Catedra de Urología. Hospital del Mar. UAB. Barcelona, España.
}

$\mathrm{E}$ 1 cáncer de Urotelio es el segundo más frecuente de los tumores genitourinarios. En Europa, alrededor de 66.000 se diagnostican de novo anualmente, si bien hay que destacar que con una irregular distribución entre países, observándose una mayor incidencia en los países del sur y de la ribera mediterránea y menos frecuente en los países del norte.

Si nos referimos a España, unos 14.000 nuevos casos se diagnostican al año. Se reconoce como la causa etiológica más importante, la que se manifiesta como diferenciada en todos los estudios epidemiológicos, el hábito de fumar.

Todos estos factores tienen una clara repercusión sobre la carga asistencial de los servicios de urología, pero también por la propia historia natural del carcinoma de urotelio, sobre la calidad de vida de los pacientes: muy frecuentes controles, cistoscopias reiteradas, riesgo de infecciones urinarias, peligro de estenosis de uretra, RTU's frecuentes con ingresos reiterados, y ello durante periodos de seguimiento largos, mínimo de unos cinco años.

Dado que la gran mayoría de los casos son no músculo-infiltrantes, la mortalidad es muy baja y al asociar la terapéutica radical precozmente cuando se detecta que se hacen infiltrantes, permite definirlo como un tumor de alta prevalencia y baja mortalidad, admitiendo que es a costa de un exhaustivo y reiterado seguimiento.

Creemos que actualmente hay aportaciones suficientes en la literatura científica que permiten vislumbrar nuevas metas asistenciales con menor morbilidad y más eficacia terapéutica.

$\mathrm{El}$ diagnóstico de recidiva en el $\mathrm{Ca}$. de Urotelio, de manera estándar, en muchos centros hospitalarios se sigue efectuando mediante la Citología (Papanicolaou) y Cistoscopia. Para el control de recidivas el inconveniente mayor de la citología es su baja sensibilidad y especificidad, así como su alto grado de subjetividad la que depende del grado de "expertise" del patólogo.

La cistoscopia sigue siendo el "gold standard", como procedimiento diagnóstico, si bien en tumores de muy pequeño volumen puede dar falsos negativos. Hay que considerarla como una prueba invasiva, a pesar de que actualmente la utilización de cistoscopios flexibles la hacen más soportable y se haya convertido en un procedimiento ambulatorio, pero no está exenta de riesgos como infección urinaria y sepsis, así como lesiones uretrales y mala visión en presencia de hematuria. Por estas razones en los últimos años han proliferado los estudios y ensayos en búsqueda de un marcador de Ca. de urotelio que no fuera invasivo, que tuviera una alta sensibilidad y alta especificidad y que la lectura de sus resultados fuera, desiderátum, totalmente objetiva.

Para el diagnóstico de tumores primarios, no existen razones de peso suficiente para sustituir totalmente a la cistoscopia, y creemos que sigue siendo imprescindible. La cistoscopia no sólo ofrece alta seguridad diagnóstica, sino que además ofrece información necesaria para la estrategia quirúrgica: volumen, topografía, relación con los meatos, número de tumores, estado del resto de la mucosa, como datos imprescindibles para planificar la cirugía, que será siempre de entrada endoscópica. En cambio referente a la citología la mayoría de kits de marcadores tumorales son más eficaces que ésta.

Pero para el seguimiento de los tumores no músculo-infiltrantes, que son afortunadamente la mayoría, hasta hace poco prácticamente no existían en la literatura estudios diseñados específicamente para responder a la pregunta de sí la cistoscopia puede o debe ser sustituida total o parcialmente en el seguimiento de tumores vesicales. Estamos frente a un dilema: por un lado la citología tiene baja sensibili- 
dad para detectar las recidivas de tumores de bajo grado y por otro lado, la cistoscopia sigue siendo un procedimiento molesto y con riesgos, y generalmente las recidivas son pequeñas, por lo cual se impone investigar nuevos marcadores y validar la seguridad de los mismos para el diagnóstico de recidiva post tratamiento endocavitario con quimio o inmunoterapia.

Actualmente existen a disposición del Urólogo una serie de kits con marcadores tumorales, unos de base genética, los que se apoyan en la técnica de FISH y otros de base proteómica, los resultados de ambos apoyan de manera clara que su fiabilidad diagnóstica puede sustituir a la citología casi siempre y en muchos casos a la cistoscopia en el seguimiento de los pacientes con $\mathrm{Ca}$. de Urotelio no músculo-infiltrante. De manera específica los basados en técnica FISH hay datos y experiencias comunicadas que apoyan su uso como método de escreening poblacional en los pacientes con factores de riesgo claramente establecido de tumor urotelial: grandes fumadores y trabajadores con exposición laboral, Lotan, Y. et als. (Cancer 2006, Sep-1, 107(5): 982-990). Por ello la FDA americana aprobó en el 2006 el escreening poblacional en grupos de riesgo mediante el uso de marcadores tumorales en orina fresca obtenida por micción espontánea, toda vez que existe un claro beneficio coste-efectividad.

Con estas herramientas nuevas se han diseñado estudios específicos para responder a la pregunta de si puede ser sustituida total o parcialmente la cistoscopia como procedimiento estándar en el seguimiento de los pacientes con cáncer de vejiga no músculo-infiltrante. En estos momentos van apareciendo resultados, unos parciales y otros definitivos, en los que parece que haciendo subgrupos de riesgo de pacientes, en algunos de ellos pueden los nuevos kits de marcadores, sustituir a la cistoscopia con una fiabilidad igual como mínimo.

Analizar los resultados de los de mayor uso y difusión que hasta el momento han aparecido en la bibliografía, así como las comunicaciones y ponencias de otros en fase de validación asistencial, creo que puede sernos útil para disponer de criterios a la hora de tomar decisiones asistenciales.

Lotan, Y. et al. en el año 2002 (J. Urol 2002, 167 (75-79) y en el 2006 (Cancer. 2006 Sep.1, 107(5): 982-990) desarrollaron un protocolo en el que proponían alternar la cistoscopia y marcadores, ello significaba una disminución del coste asistencial de estos pacientes, pero lo que era claramente diferenciado es que mejoraba los resultados. A pesar de que estos resultados son claros en su estudio, no ha tenido seguidores y no se han comunicado estudios de grandes cohortes aplicando este protocolo.

De manera clara concluyen los estudios en nuestro país el grupo de Fernández, JM. et als. (Arch. Esp. Urol, 2002, 55(10): 1201-1208) y (Comunicación al Urosimpossium-2009) afirman que el ensayo con NMP22 puede sustituir a la citología totalmente en el seguimiento ya que ofrece mayor sensibilidad, un similar VPN, y al conjugarse con la cistoscopia ofrece mayor índice de detección y, en ausencia de falsos positivos, tiene similar especificidad.

Algunos autores han propuesto nomogramas para predecir con la mayor aproximación posible las recidivas. Shariat, SF. et al. (J. Urol 2005, 173:1518-1525) desarrolló y propuso una red neuronal con tres ensayos, tres kits de marcadores, que mejoran claramente la seguridad del diagnóstico de recidiva y reducen el coste, según sus cálculos, respecto al protocolo habitual estandarizado de cistoscopia. Estos estudios, están en clara consonancia con los resultados mencionados anteriormente de Lotan, lo que confirma que si bien no permiten con estos kits, evitar totalmente la cistoscopia, si que es posible espaciarla cuando el resultado del test es negativo.

Otros estudios recientes Mansoor, I. et als. (Anal Quant Cytol Histol, 2008 Feb 30 (1):25-32) van en la línea también de que la citología en el seguimiento de pacientes, puede ser sustituida por tests como el NMP22, evitando la subjetividad interpretativa, pero que éste no puede sustituir a la cistoscopia, si bien puede ser protocolizada su alternancia en los casos en que el test es negativo; de esta manera se disminuye la morbilidad de una prueba invasiva, se disminuyen costes del seguimiento y se gana en calidad de vida del paciente en estudio.

Recientemente otros estudios se están llevando a cabo, y de los que se ha aportado resultados parciales, con test de base proteómica. En reciente comunicación parcial de resultados, Unda M. et al. (Urosimpossium-09, test Uroxact $($ ) comunicaron resultados también francamente prometedores en la tendencia a ir sustituyendo, de manera prácticamente total, la citología en el seguimiento de los tumores no músculo-infiltrantes por tests que tienen una lectura objetiva y cuya fiabilidad y VPP y VPN son iguales o ligeramente superiores. Al combinarse mediante protocolos con los controles cistoscópicos, el número de cistoscopias puede ser reducido de manera significativa, en línea con otros tests ya mencionados. 
Como primera conclusión podríamos afirmar que estos estudios demuestran que se pueden sustituir totalmente las Citología en el seguimiento de los tumores uroteliales no músculo-infiltrantes y al mismo tiempo espaciar significativamente las cistoscopias.

Existen muy pocas estrategias asistenciales en formato ensayo clínico randomizado y ciego, diseñadas específicamente para evaluar si es posible disminuir el número o sustituir totalmente la cistoscopia en el seguimiento de los tumores uroteliales no músculo-infiltrantes. Sin embargo es un objetivo asistencial en línea con la cada vez menor morbilidad en el manejo clínico de los pacientes. Sin duda las reiteradas cistoscopias en los pacientes en seguimiento, cuya fecha de interrupción de las mismas nadie se atreve a proponer con argumentos definitivos ni consensuados, ello dota de mayor necesidad, si cabe, la investigación clínica para sustituirla por procedimientos no invasivos.

Muy posiblemente los tests de base FISH, Galvan A. et al. (XII Intern. Symp. Biol. \& Clin. Useful. Of Tum. Marks. Barcelona-2009);a nálisis de Microsatélites, Zwarthoff, EC. (Scand J Urol Nephrol Suppl, 2008 Sep;(218): 147-153)); PMF-1 (factor-1 de poliamina modulado)), Aleman, A. (Clinical Cancer Resrch. 2008, Dec 15, 14(24): 8236-8243)) y el FGFR3, Cordon-Cardo, C. (Scand J Urol Nephrol Suppl 2008 Sep; (218): 154-165)), sean los mejores candidatos a plantearnos estudios prospectivos para sustituir de manera total o parcial la cistoscopia en el seguimiento de estos pacientes. De todas maneras son procedimientos que requieren una tecnología no disponible en todos los centros, personal cualificado y bien entrenado en la ejecución de estas técnicas, sin olvidar el coste del fungible, todo ello hace que hasta el momento no sean de uso generalizado.

Sin embargo algunos datos aportados a la literatura propugnan que puede ser reemplazada la Cistoscopia por alguno de estos tests ya que tanto la especificidad como la sensibilidad, así como el VPP y VPN son prácticamente coincidentes, lo que permite concluir que si bien existen falsos negativos como en la cistoscopia, en el caso de los tests, no son casos de alto grado; además con la ventaja de que con los tests se diagnostican todos los CIS, aspecto que añade mayor fiabilidad ya que la cistoscopia no los diagnostica, sólo en caso de sospecha serán las biopsias múltiples las que ofrecerán el diagnóstico.

En la misma línea de mejorar la eficacia terapéutica de la RTU y evitar las falsas recidivas por haber dejado tumor residual demostrado por RRTU, se han propugnado técnicas que identifican zonas de mucosa tumoral no objetivadas mediante las técnicas habituales con uso de luz fría o bien nidos de CIS. No referimos de manera específica a la introducción de la luz azul y el uso del ALA en la técnica habitual de resección transuretral. Hasta el momento los resultados aportados, tanto por la literatura científica internacional: Schmidbauer, J. (J Urol, Jan 171(1): 135-138, 2004), Jocham, D. (J Urol, Sep. 174 (3): 862-866, 2005), Babjuk, M. (BJU Int. Oct 96(6): 789-802, 2005) como por la bibliografía española, González, J. (Urosimpossium-09), coinciden totalmente en señalar una clara mejoría en la eficacia terapéutica, disminuyendo y casi suprimiendo el tumor residual, con lo que prácticamente desaparecen las pseudorrecidivas.

La utilización de la luz azul tiene su justificación en la terapéutica, tanto en los tumores primarios como de las recidivas, no justificándose como técnica a utilizar como escreening diagnóstico. Es decir, que en estos momentos podemos aumentar la eficacia terapéutica de la RTU de rutina mediante la combinación de la luz blanca-luz azul con administración previa de ALA en el tratamiento de los tumores de urotelio, en cifras globales del 45\%, desglosando un 38\% en los casos de CIS y 13\% en tumores Ta-T1, variando según los diferentes autores y el número de pacientes tratados. Podemos afirmar, con los datos de la literatura, que donde mayor beneficio se obtiene es cuando existe tumor de alto grado, cuando la citología es positiva y existe distancia entre las instilaciones de BCG, las que pueden dar falsos positivos.

Correspondencia autor: Dr. Antoni Gelabert Mas Servicio y Cátedra de Urología. Hospital del Mar. UAB. Po Maritim 25-29 - 08003 Barcelona. Tel.: 932483000 Información atículo: Editorial

Trabajo recibido: abril 2009 